\title{
Plasmid Profiles and Prevalence of Intermediately Virulent Rhodococcus equi from Pigs in Nakhonpathom Province, Thailand: Identification of a New Variant of the 70-kb Virulence Plasmid, Type 18
}

\author{
Chaithep Poolkhet, ${ }^{1}$ Suksun Chumsing, ${ }^{1}$ Worawidh Wajjwalku, ${ }^{1}$ Chihiro Minato, ${ }^{2}$ \\ Yukiko Otsu, ${ }^{2}$ and Shinji Takai ${ }^{2}$
}

${ }^{1}$ Faculty of Veterinary Medicine, Kasetsart University, Nakhonpathom 73140, Thailand

${ }^{2}$ School of Veterinary Medicine and Animal Science, Kitasato University, Aomori 034-8628, Japan

Correspondence should be addressed to Shinji Takai, takai@vmas.kitasato-u.ac.jp

Received 30 May 2009; Accepted 15 September 2009

Academic Editor: Takashi Umemura

Copyright (C) 2010 Chaithep Poolkhet et al. This is an open access article distributed under the Creative Commons Attribution License, which permits unrestricted use, distribution, and reproduction in any medium, provided the original work is properly cited.

\begin{abstract}
The prevalence of intermediately virulent Rhodococcus equi isolates from pig submaxillary lymph nodes from four slaughterhouses in Nakhonpathom province, Thailand, was investigated. The isolates were tested for the presence of virulence plasmids and the 20$\mathrm{kDa}$ virulence-associated protein antigen (VapB) gene by PCR. Of the 734 submaxillary lymph nodes tested, 19 (2.6\%) produced positive cultures of $R$. equi. All 19 isolates were positive for the VapB gene and contained virulence plasmids that were identified as type 1 (six isolates), type 6 (two isolates), type 7 (one isolate), type 16 (two isolates), and a new variant (eight isolates). Based on the restriction digestion patterns of the plasmid DNAs, we tentatively designated the variant as type 18. Investigation of the prevalence and plasmid profiles of VapB-positive R. equi in pigs should be extended throughout Thailand to evaluate potential sources of zoonotic infections.
\end{abstract}

\section{Introduction}

The actinomycete Rhodococcus equi is a facultative, intracellular, Gram-positive coccobacillus bacterium. Three types of $R$. equi have been classified as virulent, intermediately virulent, and avirulent on the basis of their virulenceassociated protein (Vap) antigens and virulence plasmids [1-3]. Virulent $R$. equi is characterized by the presence of the $15-17-\mathrm{kDa}$ virulence-associated protein antigen (VapA) and virulence plasmids of $85-90 \mathrm{~kb}$ and is the cause of pyogranulomatous bronchopneumonia in foals [1]. Previous studies have identified at least 12 different virulence plasmids in VapA-positive R. equi [4]. Intermediately virulent $R$. equi is characterized by the presence of a $20-\mathrm{kDa}$ virulenceassociated protein antigen (VapB) and virulence plasmids of $79-100 \mathrm{~kb}$ and can be found in pig submaxillary lymph nodes and immunocompromised patients [5]. At this time,
27 different plasmids have been identified in VapB-positive R. equi [6]. Intermediately virulent (VapB-positive) R. equi has been reported to cause fatal cavitary pneumonia because of secondary infections in immunocompromised hosts, such as AIDS patients $[7,8]$. Avirulent $R$. equi is widespread in the soil environment and is isolated predominantly from immunocompromised patients without AIDS [9].

In Thailand, infection by $R$. equi has been reported in patients with and without HIV infection in Chiang Mai, and 52 isolates from 69 sporadic cases collected between 1993 and 2001 were VapB-positive R. equi [10, 11]. Isolates of VapB-positive R. equi collected from healthy pigs at an abattoir and from immunodeficient patients in Chiang Mai contained the same plasmid types [11], so the pig was thought to be a source of VapB-positive $R$. equi infections in immunocompromised patients $[5,10,11]$. A study to examine the plasmid pattern of VapB-positive $R$. equi to 
TABLE 1: The reference strains of intermediately virulent $R$. equi with human and pig origins were used in this study.

\begin{tabular}{lc}
\hline Plasmid types & Number of reference strains \\
\hline 1 & A2 \\
2 & S2 \\
3 & S3 \\
4 & S4 \\
5 & A5 \\
6 & S6 \\
7 & S7 \\
8 & S8 \\
9 & S9 \\
10 & A11 \\
11 & A43 \\
12 & 70 \\
13 & H3 \\
14 & H25 \\
15 & H43 \\
16 & H66 \\
17 & 316 \\
\hline
\end{tabular}

evaluate any correlation between human infection and pig infection in each area was necessary to prove the source of infection. This study aimed to improve our knowledge of intermediately virulent $R$. equi in Thailand by investigating the prevalence and plasmid types of VapB-positive R. equi in the submaxillary lymph nodes of pigs in the central Thailand province of Nakhonpathom

\section{Materials and Methods}

2.1. Bacterial Strains. Seventeen representative strains of intermediate virulence (human and pig origins: Table 1) were used as plasmid-type reference strains. Some of the protein profiles, plasmid characteristics, and virulence levels of these strains have been described previously $[5,7,10,11]$.

2.2. Collection of Lymph Nodes from Slaughterhouses. Submaxillary lymph nodes were removed from freshly slaughtered pigs at 4 slaughterhouses in Nakhonpathom province, Thailand, from June 2004 to October 2005, and placed in sterile dishes for transport to the laboratory.

2.3. Isolation and Identification of Intermediately Virulent $R$. equi. The lymph nodes were immersed in boiling water for 3 seconds before they were cut up finely with sterile scissors. The pieces were placed onto nalidixic acidnovobiocin-actidione (cycloheximide)-potassium tellurite (NANAT) selective medium plates, as described previously [12]. The plates were incubated at $38^{\circ} \mathrm{C}$ in an incubator for two or three days. All suspected colonies of $R$. equi were counted, and several colonies per specimen was subcultured and then identified in our laboratory (CAMP test positive, catalase positive, urease positive, nitrate reduction test positive; glucose, sucrose, maltose, and esculin were not fermented).

2.4. Isolation and Examination of Plasmid DNA. Plasmid DNA was extracted from the isolates by the modified alkaline lysis method, as described previously [5, 13]. Plasmid DNA samples were digested with restriction endonucleases EcoRI and EcoT221, and the plasmid samples were separated on $1.0 \%$ agarose gels at approximately $5 \mathrm{~V} / \mathrm{cm}$ for 2 hours, and compared with reference plasmids.

2.5. Polymerase Chain Reaction. The target gene for polymerase chain reaction (PCR) amplification was the published sequence of the VapB gene (GenBank D44469) from R. equi strain 5 . The primers were $5^{\prime}$-GACTCTTCACAAGACGGT$3^{\prime}$ and $5^{\prime}$-TAGGCGTTGTGCCAGCT- $3^{\prime}$ for the sense and antisense strands, respectively [14]. PCR amplification was performed using $20 \mu \mathrm{L}$ of the DNA preparation in a $100 \mu \mathrm{L}$ reaction that contained $20 \mathrm{mM}$ Tris- $\mathrm{HCl}\left(\mathrm{pH} 8.3\right.$ at $\left.25^{\circ} \mathrm{C}\right)$, $100 \mathrm{mM} \mathrm{KCl}, 2.5 \mathrm{mM} \mathrm{MgCl} 2,0.4 \mathrm{mM}$ of each dNTP, $1 \mu \mathrm{M}$ of each primer, and $2.5 \mathrm{U}$ of Taq DNA polymerase (Takara, Tokyo, Japan), as described previously, with some modifications [11]. The samples were amplified for 35 cycles on a thermocycler (BioRad) under the following conditions: denaturation for 90 seconds at $94^{\circ} \mathrm{C}$, annealing for 1 minute at $55^{\circ} \mathrm{C}$, and extension for 2 minutes at $72^{\circ} \mathrm{C}[2]$.

\section{Results and Discussion}

3.1. Results. VapB-positive R. equi was isolated from 19 (2.6\%) of 734 pig submaxillary lymph nodes collected from four slaughterhouses (prevalence range, $0 \%$ to $6.3 \%$ ) in Nakhonpathom province. All were confirmed to be VapBpositive R. equi by biochemical tests and PCR amplification of the VapB gene. The plasmid profiles of the 19 isolates were investigated by restriction enzyme digestion with EcoRI and EcoT221. The 19 isolates contained virulence plasmids identified as type 1 (six isolates, $0.8 \%$ ), type 6 (two isolates, $0.3 \%$ ), type 7 (one isolate, $0.1 \%$ ), type 16 (two isolates, $0.3 \%$ ), and a new variant (eight isolates, $1.1 \%$ ).

The DNA of the 17 representative plasmids and the one new type was digested with EcoT22I and examined by Southern analysis with PCR probes. The PCR products labeled with digoxigenin-11-dUTP hybridized with one of the fragments of each plasmid DNA. From these results, we tentatively designated the new plasmid type 18. Based on the restriction digestion patterns of the plasmid DNA of the new variant, we estimated the size of the new variant to be approximately $70 \mathrm{~kb}$ (Figure 1).

3.2. Discussion. This study has shown that pigs bred in Nakhonpathom province, in the central part of Thailand, have VapB-positive $R$. equi in their submaxillary lymph nodes, with a prevalence of $2.6 \%$, which is similar to that observed in Japan [5]. Previous studies demonstrated that R. equi was widespread (average prevalence 14\%) in the 


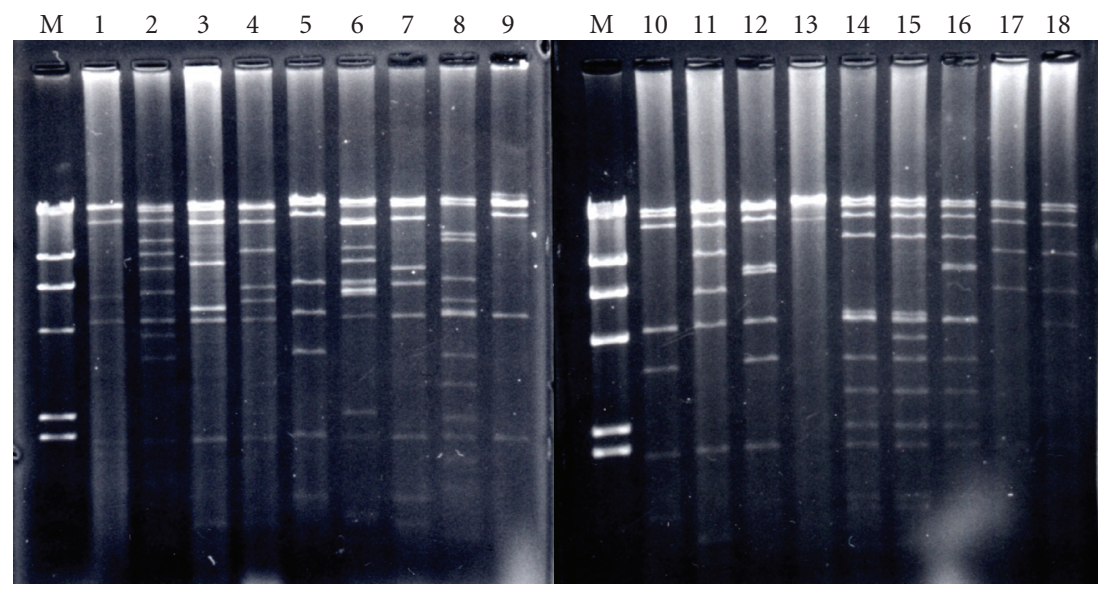

(a)

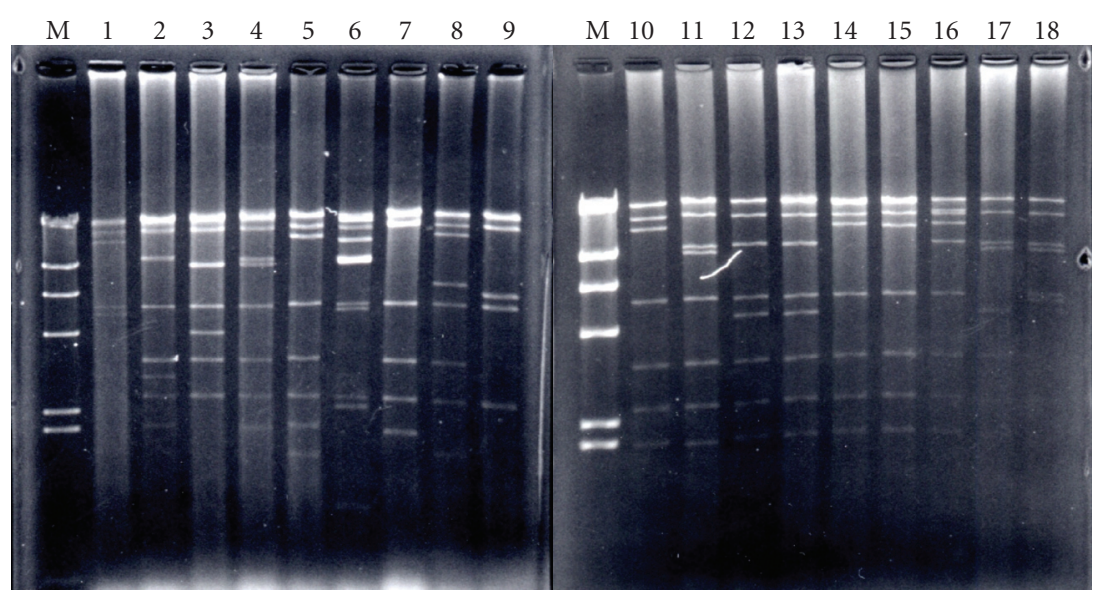

(b)

Figure 1: EcoRI (a) and EcoT22I (b) restriction fragments of the plasmid types of R. equi isolates of intermediate virulence. Lanes 1, strain A2 (plasmid type 1); lanes 2, strain S2 (plasmid type 2); lanes 3, strain S3 (plasmid type 3); lanes 4, strain S4 (plasmid type 4); lanes 5 , strain A5 (plasmid type 5); lanes 6, strain S6 (plasmid type 6); lanes 7, strain S7 (plasmid type 7); lanes 8, strain S8 (plasmid type 8); lanes 9, strain S9 (plasmid type 9); lanes 10, strain A11 (plasmid type 10); lanes 11, strain A43 (plasmid type 11); lanes 12, strain 70 (plasmid type 12); lanes 13, strain H3 (plasmid type 13); lanes 14, strain H25 (plasmid type 14); lanes 15, strain H43 (plasmid type 15); lanes 16, strain H66 (plasmid type 16); lanes 17, strain 316 (plasmid type 17); lanes 18, new strain (plasmid type 18). The markers (lanes M) are HindIII digestion products of bacteriophage $\lambda$ DNA.

submaxillary lymph nodes of domestic and wild pigs in Hungary and that the majority of $R$. equi strains from pigs were avirulent; only $25.6 \%-26.8 \%$ of the isolates in these studies were intermediately virulent (VapB-positive) and none were virulent (VapA-positive) R. equi $[6,7]$. The prevalence of intermediately virulent $R$. equi in Hungarian pigs was quite different from that demonstrated in this study or in our previous study, which showed that 368 (93.9\%) of 392 isolates collected from the submaxillary lymph nodes of Japanese pigs were intermediately virulent, whereas two $(0.5 \%)$ of the isolates were virulent and the remaining 22 (5.6\%) were avirulent [5]. Differences in the breeding of pigs in Hungary (pigs are kept in the natural environment), Thailand (pigs are kept in the backyards of farmers' houses), and Japan (pigs are kept on large-scale farms) may reflect the differences in the prevalence of each type of $R$. equi in pig isolates among these three countries.
A new variant was found in eight isolates from pigs collected at one of four slaughterhouses. Before this study, at least 18 distinct $70-100-\mathrm{kb}$ plasmids that are associated with the expression of VapB have been identified in pig and human isolates of $R$. equi $[2,5,6,11,15,16]$. However, during our study, other new types of VapB-positive have been reported by Makrai et al. (2008) [6]. Previous studies show that the immunocompromised individuals were at particular risk of infection with zoonotic diseases. $R$. equi is an emerging pathogen of humans, particularly in those with a compromised immune system [17]. Our recent studies in Thailand and Hungary have shown that the infecting $R$. equi strains are more closely related to isolates from regional pigs than other animals [7, 10,11]. Munsakul et al. reported two cases of pulmonary rhodococcosis in AIDS patients in Bangkok. There was no information concerning the source or route of infection in those two patients, such as contact 
with pigs or other domestic animals [18]. So, exploring geographic difference between AIDS patient's infection and pig's isolation in plasmid profiles of intermediately virulent $R$. equi may be a useful molecular fingerprint for epidemiological purposes to prove a route of infection in human.

The pathogenicities of these representative strains in pigs are interesting, but we do not have enough information on the pathology of the submaxillary lymph nodes of the pigs from which VapB-positive $R$. equi has been isolated. Komijn et al. (2007) investigated the prevalence of granulomatous lesions in the lymph nodes of about two million pigs in the Netherlands and isolated R. equi from 44 of 98 (44.9\%) submaxillary lymph nodes with granulomatous lesions [19]. Experimental infections in pigs showed no clinical signs other than transient fever and weight loss. Intermediately virulent strains were recovered in cultures from various organs, and the lymph nodes of pigs inoculated intravenously, but only from the mandibular lymph nodes of pigs inoculated intramuscularly [20].

\section{Conclusions}

This study showed the distribution of the plasmid profiles of VapB-positive $R$. equi isolates from healthy pigs from slaughterhouses in Nakhonpathom province, Thailand, and described a new plasmid type, type 18. This is the first report of the prevalence of VapB-positive $R$. equi in pigs in the central part of Thailand.

\section{Acknowledgments}

This study was supported by a grant from the Faculty of Veterinary Medicine, Kasetsart University, Thailand, and by a Kitasato University grant for International Exchange Programmes (2004-2006), Japan. We would also like to thank Siriluk Jala and Dr. Narin Upraragarin from the Kamphaengsean Veterinary Diagnostic Unit, Faculty of Veterinary Medicine, Kasetsart University, Thailand, for excellent technical assistance.

\section{References}

[1] S. Takai, K. Koike, S. Ohbushi, C. Izumi, and S. Tsubaki, "Identification of 15- to 17-kilodalton antigens associated with virulent Rhodococcus equi," Journal of Clinical Microbiology, vol. 29, no. 3, pp. 439-443, 1991.

[2] S. Takai, Y. Imai, N. Fukunaga, et al., "Identification of virulence-associated antigens and plasmids in Rhodococcus equi from patients with AIDS," Journal of Infectious Diseases, vol. 172, no. 5, pp. 1306-1311, 1995.

[3] O. Tkachuk-Saad and J. Prescott, "Rhodococcus equi plasmids: isolation and partial characterization," Journal of Clinical Microbiology, vol. 29, no. 12, pp. 2696-2700, 1991.

[4] M. G. Ribeiro, I. Seki, K. Yasuoka, et al., "Molecular epidemiology of virulent Rhodococcus equi from foals in Brazil: virulence plasmids of $85-\mathrm{kb}$ type I, 87-kb type I, and a new variant, 87-kb type III," Comparative Immunology, Microbiology and Infectious Diseases, vol. 28, no. 1, pp. 53-61, 2005.
[5] S. Takai, N. Fukunaga, S. Ochiai, et al., "Identification of intermediately virulent Rhodococcus equi isolates from pigs," Journal of Clinical Microbiology, vol. 34, no. 4, pp. 1034-1037, 1996.

[6] L. Makrai, A. Kobayashi, M. Matsuoka, et al., "Isolation and characterisation of Rhodococcus equi from submaxillary lymph nodes of wild boars (Sus scrofa)," Veterinary Microbiology, vol. 131, no. 3-4, pp. 318-323, 2008.

[7] L. Makrai, S. Takayama, B. Dénes, et al., "Characterization of virulence plasmids and serotyping of Rhodococcus equi isolates from submaxillary lymph nodes of pigs in Hungary," Journal of Clinical Microbiology, vol. 43, no. 3, pp. 1246-1250, 2005.

[8] Y. Mizuno, F. Sato, M. Sakamoto, et al., "VapB-positive Rhodococcus equi infection in an HIV-infected patient in Japan," Journal of Infection and Chemotherapy, vol. 11, no. 1, pp. 37-40, 2005.

[9] S. Takai, Y. Sasaki, T. Ikeda, Y. Uchida, S. Tsubaki, and T. Sekizaki, "Virulence of Rhodococcus equi isolates from patients with and without AIDS," Journal of Clinical Microbiology, vol. 32, no. 2, pp. 457-460, 1994.

[10] S. Takai, P. Tharavichitkul, C. Sasaki, et al., "Identification of virulence-associated antigens and plasmids in Rhodococcus equi from patients with acquired immune deficiency syndrome and prevalence of virulent $R$. equi in soil collected from domestic animal farms in Chiang Mai, Thailand," The American Journal of Tropical Medicine and Hygiene, vol. 66, no. 1, pp. 52-55, 2002.

[11] S. Takai, P. Tharavichitkul, P. Takarn, et al., "Molecular epidemiology of Rhodococcus equi of intermediate virulence isolated from patients with and without acquired immune deficiency syndrome in Chiang Mai, Thailand," Journal of Infectious Diseases, vol. 188, no. 11, pp. 1717-1723, 2003.

[12] J. B. Woolcock, A. M. T. Farmer, and M. D. Mutimer, "Selective medium for Corynebacterium equi isolation," Journal of Clinical Microbiology, vol. 9, no. 5, pp. 640-642, 1979.

[13] H. C. Birnboim and J. Doly, "A rapid alkaline extraction procedure for screening recombinant plasmid DNA," Nucleic Acids Research, vol. 7, no. 6, pp. 1513-1523, 1979.

[14] L. Makrai, S. Takai, M. Tamura, et al., "Characterization of virulence plasmid types in Rhodococcus equi isolates from foals, pigs, humans and soil in Hungary," Veterinary Microbiology, vol. 88, no. 4, pp. 377-384, 2002.

[15] M. Letek, A. A. Ocampo-Sosa, M. Sanders, et al., "Evolution of the Rhodococcus equi vap pathogenicity island seen through comparison of host-associated vapA and $v a p B$ virulence plasmids," Journal of Bacteriology, vol. 190, no. 17, pp. 57975805, 2008.

[16] N. Fukunaga, T. Okoda, M. Katsumi, and S. Takai, "Restriction cleavage patterns of plasmid DNA of intermediately virulent Rhodococcus equi isolates from the mandibular lymph nodes of pigs in Kagoshima, Aomori and Miyagi perfectures and the environment of pig-breeding farms," Journal of the Japan Veterinary Medical Association, vol. 52, pp. 789-792, 1999.

[17] S. Takai, "Epidemiology of Rhodococcus equi infections: a review," Veterinary Microbiology, vol. 56, no. 3-4, pp. 167-176, 1997.

[18] W. Munsakul, N. Munsakul, S. Rongjirathananont, R. Lerdpanya, N. Vititpatarapak, and L. Sangsuk, "Pulmonary rhodococcosis in AIDS patients: report of two cases in Bangkok Metropolitan Administration Medical College and Vajira Hospital," Journal of Infectious Diseases and Antimicrobial Agents, vol. 21, no. 1, pp. 25-28, 2004. 
[19] R. E. Komijn, H. J. Wisselink, V. M. C. Rijsman, et al., "Granulomatous lesions in lymph nodes of slaughter pigs bacteriologically negative for Mycobacterium avium subsp. avium and positive for Rhodococcus equi," Veterinary Microbiology, vol. 120, no. 3-4, pp. 352-357, 2007.

[20] H. Madarame, H. Matsuda, M. Okada, et al., "Cutaneous malakoplakia in pigs inoculated with Rhodococcus equi," FEMS Immunology and Medical Microbiology, vol. 22, no. 4, pp. 329333, 1998. 

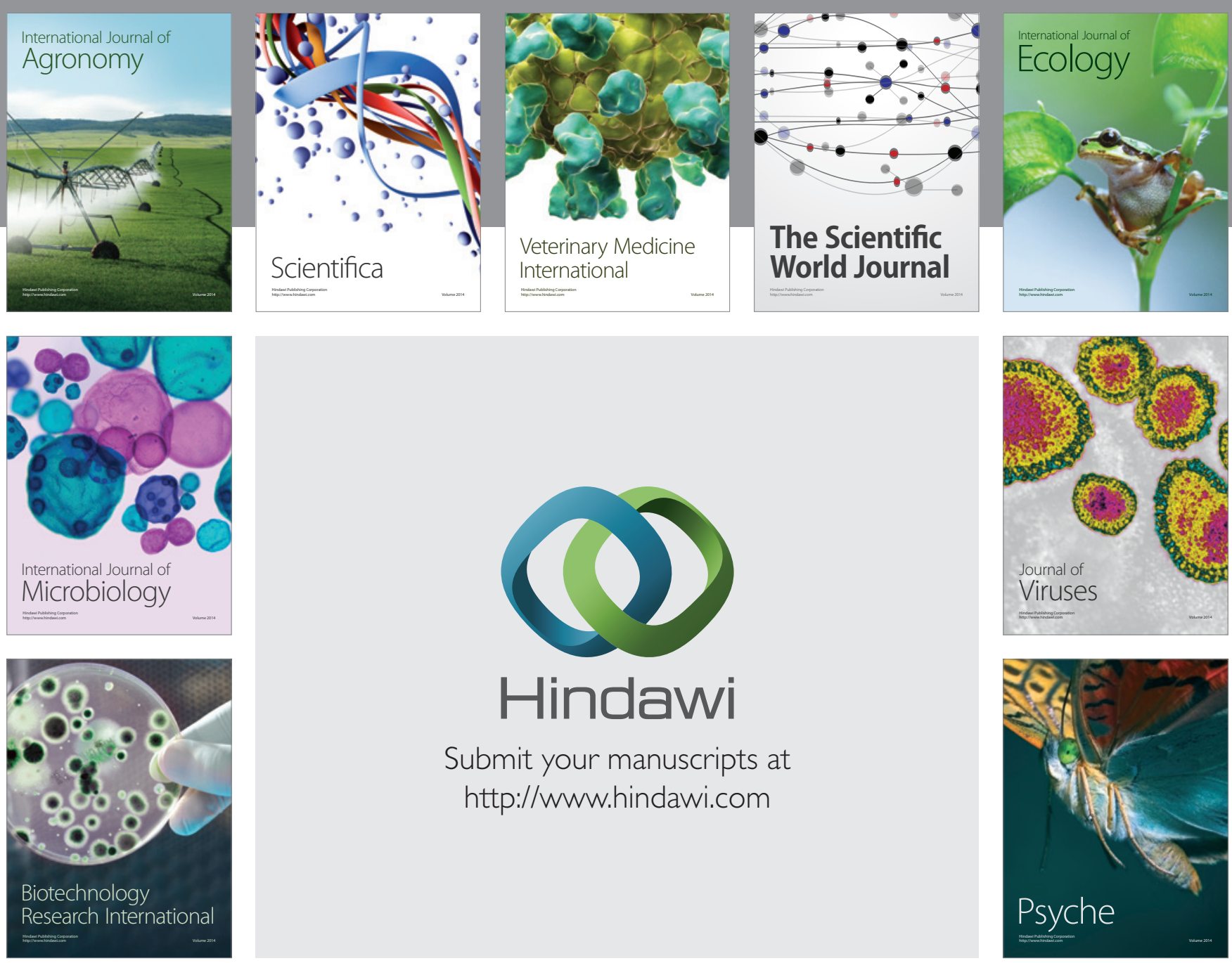

Submit your manuscripts at

http://www.hindawi.com
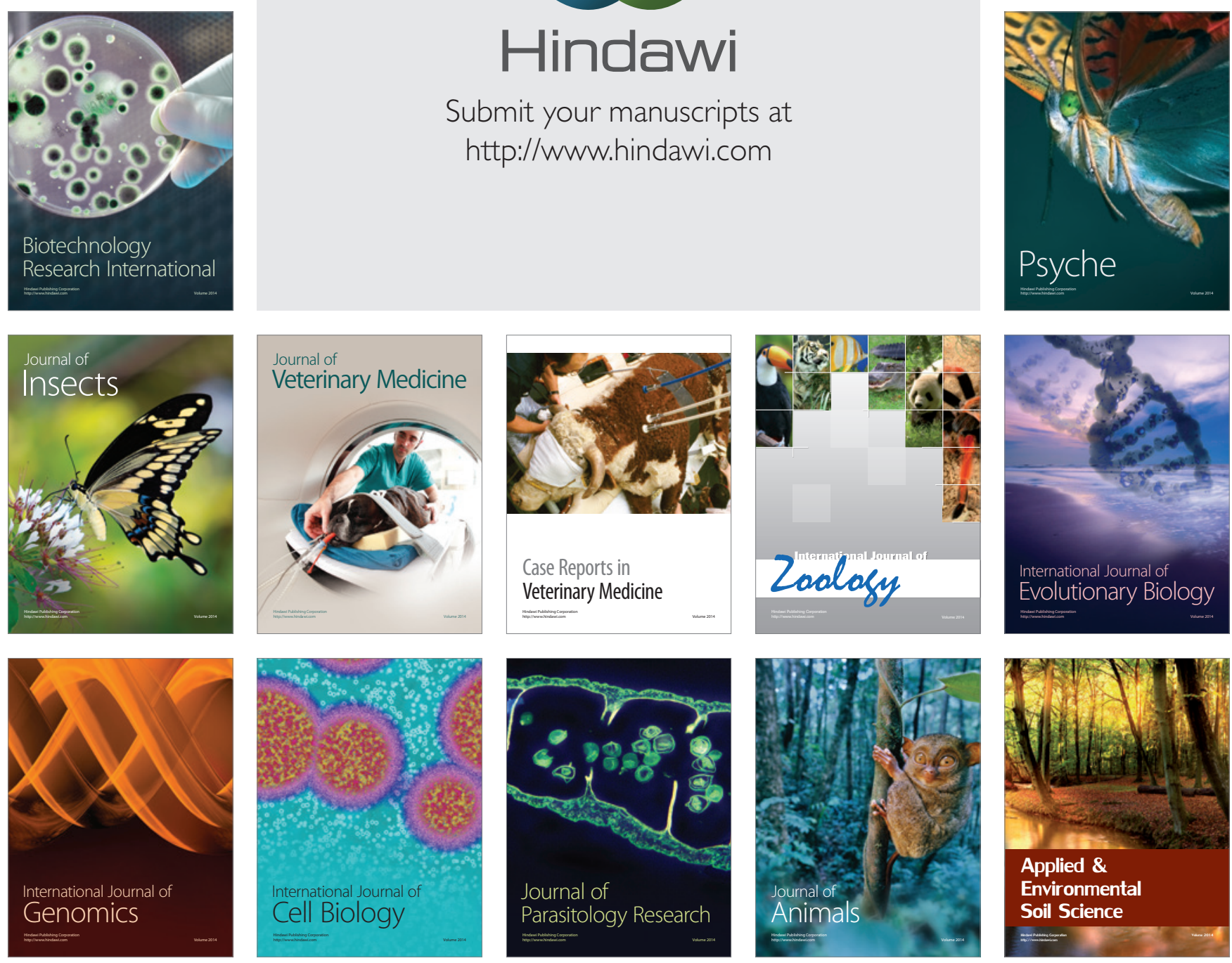